\title{
Vitamin B12-responsive methylmalonic acidemia
}

INSERM

\section{Source}

INSERM. (1999). Orphanet: an online rare disease and orphan drug data base. Vitamin B12-responsive methylmalonic acidemia. ORPHA:28

Vitamin B12-responsive methylmalonic acidemia (MA) is an inborn error of vitamin B12 (cobalamin) metabolism characterized by recurrent ketoacidotic comas or transient vomiting, dehydration, hypotonia and intellectual deficit, which responds to vitamin B12. There are three types: cblA, cblB and cblD-variant 2 (cblDv2). 